\title{
Relationship between call light use and response time and inpatient falls in acute care settings
}

\author{
Huey-Ming Tzeng and Chang-Yi Yin
}

\begin{abstract}
Aims and objectives. This exploratory study used archived hospital data to determine whether the call light use rate and the average call light response time contribute to the fall and the injurious fall rates in acute care settings.

Background. Inpatients often use call lights to seek nurses' attention and assistance. Although implied in patient safety, no studies have examined data related to the call light use or the response time to call lights collected via existing tracking mechanisms to monitor nursing practice.

Design. The study was conducted in a Michigan community hospital and used archived hospital data for analyses for the period from February 2007-June 2008. The unit of analysis was unit-week.

Method. The call light use rate per patient-day was calculated based on information retrieved from the call light tracking system. The average response time in seconds was used as generated from the tracking system. The fall and injurious fall rates per 1000 patient-days were calculated based on the fall incident reports. SPSS was used for data analyses. One-way ANOvA and correlation analyses were conducted.

Results. More calls for assistance related to less fall-related patient harm. Surprisingly, longer response time to call lights also related to fewer total falls and less fall-related patient harm. Generally speaking, more call light use related to longer response times.

Conclusions. This study's findings challenged the appropriateness of targeting the goals of reducing the frequency of call light use and the fall rates as two outcome indicators of conducting hourly patient rounds.

Relevance to clinical practice. Encouraging call light use is a key to reducing injurious fall rates. Unit managers should routinely monitor the trend of the call light use rate and ensure that the call light use rate is maintained at least above the mean rate.
\end{abstract}

Key words: accidental falls, hospitals, injuries, inpatients, safety, wounds

Accepted for publication: 9 February 2009

\section{Introduction}

Hospital inpatient falls consistently comprise the largest single category of reported accidental falls (Joint Commission 2005). Inpatient falls occur when patients fall unintentionally. For example, patients may slip, trip, or fall because of the unavailability of nurses to help when needed (e.g., delayed response to call lights). Inpatient falls can lead to injury, prolonged stays, lack of patient independence and additional resource expense (Tzeng \& Yin 2009). When inpatients need assistance (e.g., pain medication, bathroom assistance), they will often call for help verbally or use call lights to seek the nursing staff's attention. However, research on patient call light use as it pertains to patient safety during hospitalisation is limited (Meade et al. 2006, Culley 2008). The link of prompt response to patient call lights with patient
Authors: Huey-Ming Tzeng, PhD, RN, Associate Professor, Division of Nursing Business and Health Systems, School of Nursing, University of Michigan, Ann Arbor, MI, USA; Chang-Yi Yin, MA, Professor, Department of History, Chinese Culture University, Taipei, Taiwan
Correspondence: Huey-Ming Tzeng, Associate Professor, Division of Nursing Business and Health Systems, School of Nursing, University of Michigan, 400 North Ingalls, Room 4156, Ann Arbor, MI 48109, USA. Telephone: 1-734-358-0358.

E-mail: tzeng_hueyming@yahoo.com 
outcomes is often overlooked in research and practice (Deitrick et al. 2006). In addition, thus far, no studies have examined data on call light use or call light response time collected via hospitals' existing call light tracking mechanisms to monitor clinical nursing practice, which may affect patient safety outcomes. It is recognised that there are many causes of falls and the issue of falls has a multifactorial nature. Slow response to patient- or family-initiated call light is only one potential cause to falls.

\section{Purposes and rationale of this study}

This exploratory study used secondary data to investigate the correlation between the patient- or family-initiated call light use rate per patient-day and the average call light response time and the total fall and injurious fall rates per 1000 patient-days in four adult inpatient acute care units. Generally speaking, the patient- or family-initiated call light use is mainly associated with the frequency that how often patients or family visitors have unmet needs and require assistance. Response time is primarily determined by a nursing staff's reaction to each call light; the response time to each call light may be linked to the circumstance when a call goes off (e.g., the nurse-patient ratio, the acuity levels of a nurse's responsible patients). Here, it was conceptualised that the patient- or family-initiated call light use rate and the average call light response time would contribute to the total fall and injurious fall rates. This study's research question was: What are the relationships between the call light use rate and the average response time to call lights and the fall rate and injurious fall rate?

This research was conducted in a community hospital located in Michigan and used archived hospital data for analyses for the period from February 2007-June 2008 (a total of four acute adult inpatient care units). The unit of analysis was the unit-week ('unit' refers to an inpatient care unit). The goals were to generate insights about call light use and responsiveness associated with nursing service-related patient safety outcomes in inpatient care settings, that is, the total fall rate and injurious fall rate. Note that the issues of falls have a multifactorial nature, involving various risk factors as being physiological-, psychological and/or environmental-related to each individual patient (Joint Commission, 2005); there are many causes to falls other than the call light use rates and response time to call lights.

The rationale for this study arose from Donabedian's (1980) results suggesting that structure and process factors are associated with the outcome of health care organisations. In this paper, accordingly to Donabedian's (1980) model, the total fall rate and the injurious fall rate could be considered as outcome indicators associated with nursing care quality and safe hospital stays, although no studies have yet investigated the correlation of these outcome indicators with call light use and response time. Based on the reviewed studies (e.g., Curley et al. 1998, Dutton et al. 2003, Deitrick et al. 2006, Meade et al. 2006, Culley 2008), we surmised that the call light use rates and the average response time to call lights are two process indicators to predict outcome variables. Since this exploratory study was designed to explore issues related to inpatient falls from a system/macro approach by using archived hospital data/secondary data for analyses, variables included in this paper were limited by the availability of the study hospital's achieved databases. In addition, the faller and nonfaller patient profiles (e.g., risk for falls, primary medical diagnoses, acuity levels, the percentage of older patients who were cared for during the study period, mental/cognitive status and gait balance) were not included as a study limitation.

\section{Background}

\section{Nurse call systems and related issues}

Table 1 summarises selected nurse call light systems with the capability of tracking call light use. Note that all the manufacturers included are based in the USA and the products are available on the US market. The nurse call light is a vital patient communication link during hospital stays and is actually one of the few means by which patients can exercise control over their care. When patients use the call light, it is usually to summon the nurse for information or assistance. Patients expect that when they push the call light button, a nursing staff member will answer or come to them. However, the statement that call lights are perceived as noise and interruptions to nursing tasks (instead of an important way for patients to request assistance) is true according to some nurses (Meade et al. 2006).

The assumed disparity between patient and nurse perceptions of call light usage may be at the core of a patient safety problem, particularly because patients become impatient when a prompt response is not made and they attempt activities that threaten their safety. The problems associated with answering call lights also may be affecting nurse-patient communication and relationships. Communication through the call light consists of three interrelated components: (1) answering the call light, (2) communicating or responding to the patient's request and (3) following through with the request. Frustration over delays in answering call lights is one of the most frequent comments patients make. Nursing staff must recognise that call lights are legitimate ways for patients to test the responsiveness of the hospital system toward their needs (Deitrick et al. 2006). 
Table 1 Selected nurse call light systems with the capability of reporting call light use and response time*

\begin{tabular}{|c|c|}
\hline Manufacturer/product & Functions \\
\hline $\begin{array}{l}\text { Adroit Instrumentation Pte Ltd. } \\
\text { Adroit System Integration Server (2) }\end{array}$ & $\begin{array}{l}\text { Adroit System Integration Server (ASIS) was developed to increase productivity } \\
\text { and staff efficiency with an easy-to-manage and -organize software that can } \\
\text { interface with both wired and wireless nurse call systems. It automatically } \\
\text { forwards messages and keeps data of system activities for risk management } \\
\text { reports. All data are collected and processed via the ASIS and stored in the } \\
\text { application database. Managers are able to monitor and generate reports }\end{array}$ \\
\hline $\begin{array}{l}\text { Cornell Communications, Inc. } \\
\text { Visual Nurse Call Systems (6) }\end{array}$ & $\begin{array}{l}\text { Central database interface is available to provide automatic documentation } \\
\text { and recordkeeping. The Series } 4000 \text { of Visual Nurse Call Systems can } \\
\text { automatically generate a permanent record and stores all monitoring and } \\
\text { alerting activity in a historical reporting system to aid with accountability, } \\
\text { staff management, and recordkeeping }\end{array}$ \\
\hline $\begin{array}{l}\text { Intercall Systems, Inc. } \\
\text { ZbASE: Management Datebase Software (5) }\end{array}$ & $\begin{array}{l}\text { ZBASE is a management activity report package that generates customized call } \\
\text { light reports. The software is installed on a dedicated PC and operates in conjunction } \\
\text { with either a Zscan interfaced Equinox II, Legend II, or VPL300 series nurse call } \\
\text { system. It can store a complete history of calls and indicate normal, emergency, } \\
\text { and code blue calls and can generate customized reports by patient name, room } \\
\text { number and date, type of call, time of call and answer, and answering location }\end{array}$ \\
\hline $\begin{array}{l}\text { Omniwatch Technologies, Inc. } \\
\text { VisionLink Wireless Emergency Call System (4) }\end{array}$ & $\begin{array}{l}\text { The Call History software module records every resident/patient request for } \\
\text { assistance and staff response time to that request. Computer reports give the } \\
\text { number of calls and the average response times broken down by nurse station } \\
\text { and shift, a group of rooms, or a single room. The report can summarize calls by } \\
\text { time of day and the percentages that were answered in under } 3 \text { minutes, or list all } \\
\text { call activity (e.g., date, time, room number, and elapsed time to answer call). This } \\
\text { software can display call light data as a graph. Call light data can be downloaded } \\
\text { automatically }\end{array}$ \\
\hline $\begin{array}{l}\text { Systems Technologies, Inc. } \\
\text { VisionLink Wireless Nurse Call System }{ }^{\circledR}(1)\end{array}$ & $\begin{array}{l}\text { FLEXIBLE software. Patient information is a standard VisionLink }{ }^{\circledR} \text { feature along } \\
\text { with a call history log that records every resident request for assistance and staff } \\
\text { response times. Also standard are call analysis reports that provide the tools } \\
\text { toorganize and analyze the data. Additional options (as needed) are advanced } \\
\text { statistical call analysis reports }\end{array}$ \\
\hline $\begin{array}{l}\text { TekTone Sound \& Signal Mfg., Inc. } \\
\text { Tek-MMARS }{ }^{\circledR} \text { II System (3) }\end{array}$ & $\begin{array}{l}\text { The Management Monitoring and Reporting System (Tek-MMARS }{ }^{\circledR} \text { II System) is } \\
\text { an additional option for use with the Tek-CARE }{ }^{\circledR} \text { NC300 } 300^{\mathrm{TM}} \text { II Healthcare } \\
\text { Communication System. The Tek-MMARS }{ }^{\circledR} \text { II System allows production of } \\
\text { hard-copy reports, which facilitates the process of generating periodic } \\
\text { performance reports. Reporting is accessible via network }\end{array}$ \\
\hline
\end{tabular}

*All manufacturers are based in the United States, and the products are available on the US market.

The key words of 'nurse call light' and 'USA' were used to search for vendors in the Google search engine. More than 2225000 records were identified as of May 8, 2008. The first 50 records were reviewed. The relevant information was abstracted from these records.

Data sources: (1): http://www.systemstechnologiesusa.com/vlink.html; (2): http://www.adroiti.com.sg/products/Adroit\%20-\%20ASIS.html; (3): http://www.tektone.net/tekmmars2.htm; (4): http://www.omniwatchtech.com/omniinfo.htm; (5): http://www.intercallsystems.com/cat/ marspost.html; (6): http://www.cornell.com/visual-nurse-call-systems/visual.html.

\section{Call light use-related patient safety initiatives in practice}

Culley (2008) claimed that hourly nursing rounds are not a new strategy, but, in recent years, some hospitals have promoted or initiated hourly rounds to reduce the noise and interruptions caused by unnecessary call lights. Conducting hourly rounds in inpatient care units (Meade et al. 2006, Culley 2008) and the Patient Service Partner program (Gersch 1996) are studied interventions designed to decrease the frequency of call light use, reduce the fall rates and improve patient satisfaction, respectively.
For example, Meade et al. (2006) examined the impact of conducting rounds either hourly or once every two hours on the frequency of the patient call light use and patient falls. They found and categorised 26 reasons for call light use; the most common reasons were bathroom or bedpan assistance, followed by intravenous line problems or pump alarms. After implementing hourly or two-hour rounds, call light use was reduced significantly for all reasons. With one-hour rounds, there was a significant reduction in the number of falls that occurred on the units. Meade and associates' (2006) study did not address the correlation among call light use, fall rates and 
patient satisfaction scores, because these three sets of variables were investigated as the outcome indicators of the regular rounding intervention.

\section{Inpatient falls as a targeted patient safety issue}

The Joint Commission's (2008) document 'National Patient Safety Goals for 2009' continuously emphasises the need to reduce the risk of patient harms resulting from falls. The Joint Commission (2005) categorised individual risk factors for falls as either intrinsic (e.g., reduced vision, unsteady gait, musculoskeletal system deficits, mental status deficits, acute or chronic illness) or extrinsic (e.g., medication related, bed height, bedside rails, inadequate assistive devices, lack of support equipment by bathtubs and toilets, condition of floors, poor illumination) to each individual patient. Patient falls are a challenging safety and care quality issue in acute care settings, where an aging patient population or persons with physical and cognitive limitations are exposed to unfamiliar and potentially hazardous surroundings. Vulnerability may occur when patients with acute, life-threatening medical conditions first come for treatment or immediately after treatments, procedures, or surgery (Reiling 2006). Falls among people aged 65-74 are more likely to be due to extrinsic factors, whereas the intrinsic factors are more important among those older than 80 years (Hignett \& Masud 2006).

Fall prevention programs for hospitalised patients have had limited success because patients have increasingly complex disorders and functional deficits and staff members often fail to consider pertinent risks such as a patient's tendency to overstep physical limitations (Jeske et al. 2006). Recent metaanalyses of hospital fall intervention studies have found: (1) limited research that adequately evaluates recommendations or programs (Hignett \& Masud 2006); (2) lack of reproducibility of interventions within and among different settings; and (3) lack of evidence of cost effectiveness of interventions (Oliver et al. 2007). To design a sustainable fall prevention program, crucial precedents and objective factors of the fall and fall injury rates must first be identified through research (e.g., call light use and response time).

\section{Call light use and inpatient falls}

An exploratory study conducted at a Michigan hospital identified the extrinsic risk factors for inpatient falls based on nurse and nursing attendant interviews. Several human resource issues were found as related to call light use, including difficulties in determining patient care priorities, nursing staff's misconception about the purpose of call lights, call lights not being answered in a timely manner, difficulty in implementing timed observation and toileting plans and patient assignments not being in close proximity, which may result in delays in responding to patients' call lights and needs (Tzeng \& Yin 2008a). Another study explored the potential solutions to eliminate the extrinsic risk factors that may contribute to inpatient falls from the perspectives of nurses (Tzeng \& Yin 2008 b). Solutions related to call light use included improving teamwork and responsibility sharing among nurses and nursing attendants on answering the call lights as quickly as possible, making rounds to ensure that the patients are contented and their needs are met and ensuring continual observation.

Tzeng and Yin (2009) conducted a study at a Michigan hospital to understand the opinions and observations of recently discharged older adult patients about the fall prevention program during their most recent hospitalisation. Lack of availability of nurses to help when needed (e.g., delayed response to call lights) was indicated as one of the main safety concerns. Patients or their families emphasised that nurses should monitor patients constantly and provide assistance and answer a call light in a timely manner.

\section{Methods}

\section{Design}

This research was conducted in a community hospital located in Michigan. This project was approved by the institutional review board of the study hospital and the employed university. It used archived hospital data for the period between 5 February 2007-29 June 2008 for a secondary data analysis (a total of 72 weeks). This project explored the contribution of the call light use rate and the average response time to the fall rate, the injurious fall rate and patient satisfaction scores that occurred in four adult inpatient acute care units. Among these four units, two were medical units (a total of 87 beds), one surgical unit (53 beds) and one medical-surgical combined unit (58 beds). The unit of analysis was the unit-week; a total of 288 unit-week data points were available for analyses.

\section{Data source and collection}

\section{Call light data}

The information recorded from the patient room call light system was used in this study. It refers to the call light button, which is attached to the patient room wall, above the headboard of the bed. The patient room call light system can be used when a patient is lying on the bed or sitting on a bedside chair or commode. 
The call light use rate per patient-day was calculated based on the information retrieved from the call light tracking system. The total call light use counts by unit-week were generated. A week started on Monday and ended on Sunday. Note that occasionally the call light tracking program may fail to record call light use and response time because of power outage and equipment failure. Consequently, when generating a call light report, the number of the covered days is automatically indicated. The information on the number of the covered days was used in this study for justifying the count of the total call light use for the defined period. The computation for the call light rate by unit-week was: (the counts of the total call light use/the number of the covered days) $\times$ seven days/the total patient-days for the week. The average response time in seconds was used as generated from the call light tracking system.

\section{Fall data}

The rate of inpatient falls, which have long been perceived as a nursing-sensitive quality indicator, is defined as the rate at which patients fall during their hospital stays per 1000 patient-days (American Nurses Association 2002). Falls include those with no apparent injury, those with minor, moderate, or major injuries, or those resulting in death. Injurious falls refer to ones with moderate or major injuries or resulting in death. Total fall counts and the severity level of these falls were requested from the study hospital. No falls resulted in death in the study units during the study period.

The computation for the fall rate by unit-week was: (the counts of all patient falls/the total patient-days for the week) $\times 1000$ patient-days. The computation for the injurious fall rate by unit-week was: (the counts of injurious falls/ the total patient-days for the week) $\times 1000$ patient-days.

\section{Data analyses}

Collected data were entered and processed using SPSS statistical software. One-way ANOva was used to compare the differences across three unit types on the means of the call light use rate, the average call light response time, the fall rate and the injurious fall rate. Pearson and Spearman's rho correlation analyses were used to explore the relationships between the call light use rate and the average call light response time and the identified patient outcome variables (the fall rate and the injurious fall rate). Correlation analyses were conducted on the weekly unit-level data for all available unit-week data points and on the data points by three unit types (medical, surgical and medical-surgical combined units). Alpha was set at 0.05.

\section{Results}

Table 2 illustrates the descriptive information of the study variables by unit types and for all available unit-level data points $(n=288)$. The findings of one-way ANOva tests on the mean values of the study variables across three unit types are also summarised in Table 2. The one-way ANOva and Tukey significant difference tests showed that statistically significant differences occurred in the mean values across the medical, surgical and medical-surgical combined unit data on the call light use rate; the mean values ranged from 3.606.08 counts per patient-day. For the average call light response time, the mean difference between medical and surgical unit data was statistically significant; the mean values ranged from three minutes 2.86 seconds-three minutes 30.72 seconds. For the fall rates, the mean difference between medical-surgical combined and surgical unit data was statistically significant.

The Pearson correlation analyses (Table 3 ) showed that for the medical unit data, when the call light use rate was higher, the average call light response time was longer $(r=0 \cdot 41$, $p<0.01)$; when using all data points, the correlation coefficient was 0.37 ( $p<0.01)$. When using all data points, when the average call light response time was higher, the fall rate was lower $(r=-0 \cdot 15, p<0 \cdot 01, n=288)$. For the medical-surgical combined unit data, when the call light use rate was higher, the injurious fall rate was lower $(r=-0.35$, $p<0.01, n=288)$. When the average call light response time was higher, the injurious fall rate was lower $(r=-0 \cdot 27$, $p=0 \cdot 02, n=288$ ).

The Spearman's rho correlation analyses (Table 4) indicated that that for the medical unit data, when the call light use rate was higher, the average call light response time was longer $(r=0.42, p<0.01)$; when using all data points, the correlation coefficient was $0.55(p<0.01)$. When using all data points, when the call light use rate was higher, the injurious fall rate was lower $(r=-0 \cdot 12, p=0 \cdot 04, n=288)$. When the average call light response time was higher, the fall rate was lower $(r=-0 \cdot 16, p<0 \cdot 01, n=288)$. For the medical unit data, when the average call light response time was higher, the fall rate was lower $(r=-0 \cdot 16, p=0 \cdot 05$, $n=288$ ). For the medical-surgical combined unit data, when the call light use rate was higher, the injurious fall rate was lower $(r=-0.32, p<0 \cdot 01, n=288)$.

\section{Discussion}

This exploratory study examined the relationships among the call light use rate and the average response time to call lights and the fall rate and injurious fall rate in four adult inpatient 
Table 2 Descriptive characteristics of the study variables and the summary of the one-way ANOva results about the differences across three unit types on the means of the study variables

\begin{tabular}{|c|c|c|c|c|c|}
\hline Variable & Unit type & Mean & SD & Minimum-maximum & $\begin{array}{l}\text { One-way ANOVA } \\
\text { F value (significance) }\end{array}$ \\
\hline \multirow{4}{*}{$\begin{array}{l}\text { Call light use rate } \\
\text { per patient-day }\end{array}$} & Medical $(n=144)$ & $3 \cdot 60$ & 1.91 & $0 \cdot 32-11 \cdot 57$ & $F=51.80^{* *}(p=0.00)$ \\
\hline & Surgical $(n=72)$ & $6 \cdot 08$ & $1 \cdot 45$ & $0 \cdot 65-7 \cdot 99$ & \multirow{3}{*}{$\begin{array}{l}\text { The means of these three types } \\
\text { of units were statistically } \\
\text { significantly different from } \\
\text { each other (Tukey test }{ }^{\dagger}, \\
p<0.01 \text { ) }\end{array}$} \\
\hline & Med-surgical $(n=72)$ & $4 \cdot 43$ & $1 \cdot 39$ & $0 \cdot 74-6.99$ & \\
\hline & Total $(n=288)$ & $4 \cdot 41$ & $1 \cdot 96$ & $0 \cdot 32-11 \cdot 57$ & \\
\hline \multirow{4}{*}{$\begin{array}{l}\text { Average call light response } \\
\text { time in seconds }\end{array}$} & Medical $(n=144)$ & $182 \cdot 86$ & 88.98 & $58-391$ & $F=4 \cdot 16^{*}(p=0.02)$ \\
\hline & Surgical $(n=72)$ & $210 \cdot 72$ & $25 \cdot 74$ & $162-280$ & \multirow{3}{*}{$\begin{array}{l}\text { The mean difference between } \\
\text { the medical unit data and the } \\
\text { surgical unit data was } \\
\text { statistically significant (Tukey } \\
\text { test }^{\dagger}, p=0.01 \text { ) }\end{array}$} \\
\hline & Med-surgical $(n=72)$ & $195 \cdot 07$ & $37 \cdot 54$ & $98-277$ & \\
\hline & Total $(n=288)$ & $192 \cdot 78$ & $67 \cdot 92$ & $58-391$ & \\
\hline \multirow{4}{*}{$\begin{array}{l}\text { Total fall rate } \\
\text { per } 1000 \text { patient-days }\end{array}$} & Medical $(n=144)$ & $3 \cdot 76$ & $4 \cdot 23$ & $0 \cdot 00-17 \cdot 34$ & \multirow{4}{*}{$\begin{array}{l}F=4 \cdot 88^{* *}(p=0 \cdot 01) \\
\text { The mean difference between } \\
\text { the medical-surgical } \\
\text { combined unit data and the } \\
\text { surgical unit data was } \\
\text { statistically significant } \\
\left.\text { (Tukey test }{ }^{\dagger}, p<0 \cdot 01\right)\end{array}$} \\
\hline & Surgical $(n=72)$ & $2 \cdot 68$ & $3 \cdot 58$ & $0 \cdot 00-14 \cdot 22$ & \\
\hline & Med-surgical $(n=72)$ & $4 \cdot 74$ & $3 \cdot 71$ & $0 \cdot 00-16 \cdot 29$ & \\
\hline & Total $(n=288)$ & $3 \cdot 74$ & $4 \cdot 00$ & $0 \cdot 00-17 \cdot 34$ & \\
\hline \multirow{4}{*}{$\begin{array}{l}\text { Injurious fall rate } \\
\text { per } 1000 \text { patient-days }\end{array}$} & Medical $(n=144)$ & $0 \cdot 17$ & $0 \cdot 85$ & $0 \cdot 00-5 \cdot 81$ & \multirow[t]{4}{*}{$F=0.53(p=0.59)$} \\
\hline & Surgical $(n=72)$ & $0 \cdot 07$ & $0 \cdot 56$ & $0 \cdot 00-4 \cdot 74$ & \\
\hline & Med-surgical $(n=72)$ & $0 \cdot 18$ & $0 \cdot 75$ & $0 \cdot 00-3 \cdot 44$ & \\
\hline & Total $(n=288)$ & $0 \cdot 15$ & $0 \cdot 76$ & $0 \cdot 00-5 \cdot 81$ & \\
\hline
\end{tabular}

The unit of analysis is unit-week.

$* p<0 \cdot 05, * p<0 \cdot 01$.

†Tukey honestly significant difference test, which produces multiple comparisons among all groups.

Table 3 Summary of the Pearson correlation analyses between the call light use rate and the call light response time and the fall rate and injurious fall rate

\begin{tabular}{|c|c|c|c|c|c|c|c|c|}
\hline $\begin{array}{l}\text { Unit type/study } \\
\text { variable/Pearson } \\
\text { coefficient } \\
\text { (significance) }\end{array}$ & $\begin{array}{l}\text { All data points } \\
(n=288)\end{array}$ & & $\begin{array}{l}\text { Medical unit } \\
\text { data }(n=144)\end{array}$ & & $\begin{array}{l}\text { Surgical unit } \\
\text { data }(n=72)\end{array}$ & & $\begin{array}{l}\text { Medical-surgica } \\
\text { unit data }(n=7\end{array}$ & $\begin{array}{l}\text { l combined } \\
\text { 2) }\end{array}$ \\
\hline $\begin{array}{l}\text { Call light use rate } \\
\text { per patient-day } \\
\text { (abbreviated } \\
\text { as call light use) }\end{array}$ & $\begin{array}{l}\text { Call light } \\
\text { use }\end{array}$ & $\begin{array}{l}\text { Response } \\
\text { time }\end{array}$ & $\begin{array}{l}\text { Call light } \\
\text { use }\end{array}$ & $\begin{array}{l}\text { Response } \\
\text { time }\end{array}$ & $\begin{array}{l}\text { Call light } \\
\text { use }\end{array}$ & $\begin{array}{l}\text { Response } \\
\text { time }\end{array}$ & $\begin{array}{l}\text { Call light } \\
\text { use }\end{array}$ & $\begin{array}{l}\text { Response } \\
\text { time }\end{array}$ \\
\hline $\begin{array}{l}\text { Average call light } \\
\text { response time } \\
\text { (abbreviated as } \\
\text { response time) }\end{array}$ & $0 \cdot 37 *(0 \cdot 00)$ & - & $0 \cdot 41 *(0 \cdot 00)$ & - & $0.02(0.86)$ & - & $0 \cdot 21(0 \cdot 08)$ & - \\
\hline $\begin{array}{l}\text { Total fall rate } \\
\text { Injurious fall rate }\end{array}$ & $\begin{array}{l}-0.09(0.14) \\
-0.11(0.07)\end{array}$ & $\begin{array}{l}-0 \cdot 15 *(0 \cdot 01) \\
-0 \cdot 10(0 \cdot 10)\end{array}$ & $\begin{array}{l}-0.05(0.59) \\
-0.05(0.54)\end{array}$ & $\begin{array}{l}-0.15(0.07) \\
-0.07(0.39)\end{array}$ & $\begin{array}{r}-0.00(0.98) \\
0.08(0.54)\end{array}$ & $\begin{array}{l}-0.09(0.45) \\
-0.00(0.98)\end{array}$ & $\begin{array}{l}-0.05(0.68) \\
-0.35 *(0.00)\end{array}$ & $\begin{array}{l}-0 \cdot 19(0 \cdot 12) \\
-0 \cdot 27^{*}(0 \cdot 02)\end{array}$ \\
\hline
\end{tabular}

The unit of analysis is unit-week.

$* p<0 \cdot 05, * p<0 \cdot 01$.

acute care units. As indicators, the call light use rate and the average call light response time reflect the reality of patients' hospitalisation experience and provide an overall under- standing of the patterns of an inpatient unit's care delivery. This study illustrates that, generally, more call light use related to longer response time. More calls for assistance 
Table 4 Summary of the nonparametric (Spearman's rho) correlation analyses between the call light use rate and the call light response time and the fall rate and injurious fall rate

\begin{tabular}{|c|c|c|c|c|c|c|c|c|}
\hline $\begin{array}{l}\text { Unit type/study } \\
\text { variable/Spearman } \\
\text { rho coefficient } \\
\text { (significance) }\end{array}$ & $\begin{array}{l}\text { All data points } \\
(n=288)\end{array}$ & & $\begin{array}{l}\text { Medical unit } \\
\text { data }(n=144)\end{array}$ & & $\begin{array}{l}\text { Surgical unit } \\
\text { data }(n=72)\end{array}$ & & $\begin{array}{l}\text { Medical-surgica } \\
\text { unit data }(n=7\end{array}$ & $\begin{array}{l}\text { al combined } \\
72 \text { ) }\end{array}$ \\
\hline $\begin{array}{l}\text { Call light use rate } \\
\text { per patient-day } \\
\text { (abbreviated } \\
\text { as call light use) }\end{array}$ & $\begin{array}{l}\text { Call light } \\
\text { use }\end{array}$ & $\begin{array}{l}\text { Response } \\
\text { time }\end{array}$ & $\begin{array}{l}\text { Call light } \\
\text { use }\end{array}$ & $\begin{array}{l}\text { Response } \\
\text { time }\end{array}$ & $\begin{array}{l}\text { Call light } \\
\text { use }\end{array}$ & $\begin{array}{l}\text { Response } \\
\text { time }\end{array}$ & $\begin{array}{l}\text { Call light } \\
\text { use }\end{array}$ & $\begin{array}{l}\text { Response } \\
\text { time }\end{array}$ \\
\hline $\begin{array}{l}\text { Average call light } \\
\text { response time } \\
\text { (abbreviated } \\
\text { as response time) }\end{array}$ & $0 \cdot 42 *(0.00)$ & - & $0.55 *(0.00)$ & - & $0.08(0.51)$ & - & $0.01(0.96)$ & - \\
\hline Total fall rate & $-0.08(0.19)$ & $-0 \cdot 16^{* *}(0 \cdot 01)$ & $-0.07(0.40)$ & $-0 \cdot 16^{*}(0.05)$ & $-0.02(0.89)$ & $-0.02(0.86)$ & $0.06(0.63)$ & $-0 \cdot 18(0 \cdot 14)$ \\
\hline Injurious fall rate & $-0 \cdot 12 *(0 \cdot 04)$ & $-0.08(0 \cdot 18)$ & $-0.03(0.72)$ & $-0.05(0.58)$ & $0 \cdot 12(0 \cdot 31)$ & $0.01(0.92)$ & $-0 \cdot 32 * *(0 \cdot 01)$ & $-0 \cdot 17(0 \cdot 16)$ \\
\hline
\end{tabular}

The unit of analysis is unit-week.

$* p<0.05, * p<0 \cdot 01$.

related to less fall-related patient harm in the adult inpatient acute care settings of the study hospital. Surprisingly, longer response time to call lights also related to fewer total falls and less fall-related patient harm.

It is arguable that encouraging call light use is a key to reducing injurious fall rates. We suspected that the organisational culture and leadership style of the study hospital (e.g., the chief nurse's expectations on nurses' attitudes and behaviors toward patient care services and the leadership style of the unit managers) may have influence on how nursing staff respond to call lights. Note that in the study units, the call light console is located in the nurses' station. Nursing staff must pay attention to the signal system located next to the door of the patient room regularly to learn patients' needs if any. Otherwise, the responsible nursing staff will be notified via the overhead speakers by the unit clerk who answers the call light at the nurses' station. As we have observed, most of the nurses, who work in the study units, tend to stay nearby their assigned patients (e.g., charting on the close by satellite computers). According to some nurses working in the study hospital, they have been instructed by their nurse managers to put patients' needs first and respond to call lights in a minute if possible. Often, if a call goes off, the responsible nurse will attend to his or her patient's needs first and then cancel the call light after completing the needed tasks. If so, longer response time to call lights may suggest that the responsible nurse spend more time at bedside (translated into more bedside nursing hours). More bedside nursing hours per patient day may consequently result in safer hospital stays (e.g., fewer total falls and less fall-related patient harm).
Note that this study did not address nurses' judgment or behaviors related to answering call lights. Nevertheless, it should also be noted that nurses may not respond to a call light with similar speed among the patients with the same diagnoses or medical problems based on their assessment of patients' conditions that day. Consequently, more research is needed to explore the expected response time to patient- and family-initiated call lights as perceived by the chief nurse, unit managers and nursing staff and patients and their family visitors. It would also be essential to investigate the relationship between the expected response time by nursing staff and unit managers and the average response time to call lights as documented by the call light tracking system and the relationship between the response time and nursing staff' perceived organisational culture of their working hospital and their behaviors associated with the safety culture in their working units.

In addition, we perceived that the call light use rate per patient-day reflects the frequency of requests for assistance and these calls may be placed by patients or family visitors. Hospital-paid sitters, who provide continuous observation of patients, may also initiate the calls, although sitters are not used routinely at the study hospital. According to some nurses, it is true that the average response time to call lights reveals nursing staff's reactions to patients and patientinitiated call lights, which may be associated with their judgment and perceived urgency levels of the calls, their action capability and promptness (e.g. retrieving needed equipment) and the business level of their working unit (e.g. number of new admissions and patients who require preprocedure or operation preparation). 


\section{Limitations}

As for the study limitations, the call light tracking system adopted in the study hospital and the ones available on the market do not yet differentiate whether a call light was being responded to or was cancelled at the bedside or by the console located in the nurses' station. In addition, the tracking system cannot distinguish whether the response to a call light occurs before or after the reason for this call light is resolved and whether nursing staff resolves the reason for this call light effectively.

This study investigated only the information recorded from the patient room call light system. All call lights from this call light button have the same beeping sound indicated on the console located in the nurses' station; they all show the same signal light and blinking style on the signal system located next to the door of the patient room and connected to the call light system. Thus, all the calls analysed in this study showed the same urgency level; the call light system does not have the capability to tell nursing staff the urgency level of each call light. Nurses must go into a patient's room or communicate with the patient or family visitors via intercom to determine the urgency level of each call. The information recorded by the call light systems in the bathrooms was not the focus of this study and should be investigated in future research.

Obviously, a nurse cannot physically attend to more than one patient's call at the same time. A call light may be answered by a registered nurse, a nurse aide, a unit clerk at the nurses' station, or other health care providers (e.g., physical therapists). Consequently, we assume that the efficiency of the teamwork among a patient's responsible staff may contribute to a patient's willingness to use the nurse call system to request assistance and the response time to each call light. In addition, whether the call light button is placed at a convenient location for the patient may also be factor in the use rate. Nursing staff's priority among their assigned tasks (e.g., answering call lights, preparing for medication administration, retrieving supplies and writing documentation), patients' acuity levels and changes in their patients' physical conditions and mental status (e.g., being confused, feeling depressed and lonely) may also be critical factors to their response time to call lights. These issues are not addressed and were not able to be controlled in this paper as study limitations.

\section{Conclusions}

In practice, the patient- or family-initiated call light use rate and the average staff response time to call lights are well recognised as two indicators to reflect the reality of patients' hospitalisation experiences and provide an overall understanding of the patterns of an inpatient care unit's care delivery. Based on the findings of this study, it is logical to suggest that the call light use rate per patient-day is a predictor of the fall and injurious fall rates. Consequently, unit managers should routinely monitor the trend of the call light use rate per patient-day and ensure that this use rate is maintained at least above the mean rate. As a matter of fact, this study's findings challenge the appropriateness of targeting the goals of reducing the frequency of call light use and the fall rates as two outcome indicators of conducting hourly rounding in inpatient care units (Meade et al. 2006, Culley 2008) and the Patient Service Partner program (Gersch 1996). Multihospital studies are needed to demonstrate whether the same conclusion holds across hospitals. Practical implications and future research directions are illustrated below.

\section{Relevance to clinical practice and future research directions}

Based on the results of one-way ANOVA and correlation analyses (Tables 2-4), it would be more practical to interpret the findings by unit types (medical, surgical and medicalsurgical combined units), rather than treating the data from three different unit types equally. Consequently, strategies to prevent fall and injurious falls should be proposed based on the unit types.

Future research should investigate the differences across unit types by introducing unit-level indicators, such as human resource characteristics (e.g., total nursing hours and total registered nurse hours per patient-day, scheduling patterns, average patient acuity and mobility levels). It is also essential to conduct multihospital research to discern the differences across different types of hospitals. If so, hospital-level indicators (e.g., principles in staffing mix, being a magnet hospital and organisational culture) should be considered when verifying the relationship between the call light use rate and the average call light response time and fall and injurious fall rates.

As we have observed in practice, the reasons for patientinitiated call lights are primarily for pain medications, intravenous problems or pump alarms, or bathroom, bedside commode, or bedpan assistance. It is arguable that most of the patients' problems are physical. Consequently, we conclude that encouraging call light use is a key to reducing injurious fall rates. For nursing executives, it is essential to routinely monitor the trend of the call light use rate per patient-day and to ensure that the call light use rate is maintained at least above the mean rate. 
A downward trend of call light use rates may lead to higher injurious fall rates because of the lack of proper actions of the nursing staff (e.g., failing to place the nurse call button within patients' reach). Nursing staff should be educated to treat all call lights initiated from the patient room call system equally and this guideline should be reiterated regularly as one of the patient safety initiatives to promote safer hospital stays. However, these statements need further research for validation.

The design of the call button (e.g., the size of the nurse call button and the length of the connecting cord) should be evaluated to understand whether the nurse call button is easily located, grasped and operated by patients whey they are lying in bed. When purchasing the nurse call button, nurse executives and unit managers should consider that both geriatric and non-geriatric patients may encounter difficulties in locating the right button to push for assistance because of poor vision and clumsy finger movements (e.g., swollen fingers due to arthritis). A simplified call light button, which does not have the remote television and radio control functions on the same panel, can be the answer to promote call light use.

More research is needed to explore the differences in the reasons for patient-initiated call lights between surgical and medical patients. It is important to explore the differences in the reasons for patient- and family-initiated call lights as perceived by nursing staff who work in different types of inpatient care units. After cross-validating the call light tracking data with the perspectives of nursing staff and patients and family visitors toward patient- and familyinitiated call lights, interventions may be designed to promote safer hospital stays and improve both patient satisfaction and nurses' morale.

\section{Acknowledgement}

Sources of support: Not applicable.

\section{Contributions}

Study design: H-MT; data collection and analysis: H-MT, $\mathrm{C}-\mathrm{YY}$ and manuscript preparation: H-MT.

\section{References}

Culley T (2008) Reduce call light frequency with hourly rounds. Nursing Management 39, 50-51.
Curley C, McEachern JE \& Speroff T (1998) A firm trial of interdisciplinary rounds on the inpatient medical wards: an intervention designed using continuous quality improvement. Medical Care 36(8 Suppl), AS4-AS12.

Deitrick L, Bokovoy J, Stern G \& Panik A (2006) Dance of the call bells: using ethnography to evaluate patient satisfaction with quality of care. Journal of Nursing Care Quality 12, 316-324.

Donabedian A (1980) Explorations in quality assessment and monitoring. The Definition of Quality and Approaches to Its Assessment, Vol. I. Health Administration Press, Ann Arbor, MI.

Dutton RP, Cooper C, Jones A, Leone S, Kramer ME \& Scalea TM (2003) Daily multidisciplinary rounds shorten length of stay for trauma patients. Journal of Trauma 55, 913-919.

Gersch P (1996) Initiating a patient service partner program. Nursing Management 27, 46-50.

Hignett S \& Masud T (2006) A review of environmental hazards associated with in-patient falls. Ergonomics 49, 605-616.

Jeske L, Kolmer V, Muth M, Cerns S, Moldenhaur S \& Hook ML (2006) Partnering with patients and families in designing visual cues to prevent falls in hospitalized elders. Journal of Nursing Care Quality 21, 236-242.

Joint Commission (2005) Defining the problem of falls. In Reducing the Risk of Falls in Your Health Care Organization (Smith IJ, ed.). Joint Commission, Oakbrook Terrace, IL, pp. 13-27.

Meade CM, Bursell AL \& Ketelsen L (2006) Effects of nursing rounds on patients' call light use, satisfaction and safety. American Journal of Nursing 106, 58-70.

Oliver D, Connelly JB, Victor CR, Shaw FE, Whitehead A, Genc Y, Vanoli A, Martin FC \& Gosney MA (2007) Strategies to prevent falls and fractures in hospitals and care homes and effect of cognitive impairment: systematic review and meta-analyses. BMJ $334,82$.

Reiling J (2006) Safe design of healthcare facilities. Quality and Safety in Health Care 15(Suppl. 1), i34-i40.

Tzeng HM \& Yin CY (2008a) The extrinsic risk factors for inpatient falls in hospital patient rooms. Journal of Nursing Care Quality 23, 234-242.

Tzeng HM \& Yin CY (2008b) Nurses' solutions to prevent inpatient falls in hospital patient rooms. Nursing Economics 26, 179-187.

Tzeng HM \& Yin CY (2009) Perspectives of recently discharged patients on hospital fall-prevention programs. Journal of Nursing Care Quality 24, 42-49.

\section{Online References}

American Nurses Association (2002) Quality Indicators: Outcomes Measurement Using the ANA Safety and Quality Indicators. http:// nursingworld.org/mods/archive/mod72/ceomfull.htm (accessed 17 June 2009).

Joint Commission (2008) 2009 National Patient Safety Goals: Hospital Program. http://www.jointcommission.org/PatientSafety/ NationalPatientSafetyGoals/09_hap_npsgs.htm (accessed 9 August 2008). 